Oscillatory dynamics of an intravenous glucose tolerance test model with delay interval

Xiangyun Shi, Yang Kuang, Athena Makroglou, Sriprakash Mokshagundam, and Jiaxu Li

Citation: Chaos 27, 114324 (2017);

View online: https://doi.org/10.1063/1.5008384

View Table of Contents: http://aip.scitation.org/toc/cha/27/11

Published by the American Institute of Physics 


\title{
Oscillatory dynamics of an intravenous glucose tolerance test model with delay interval
}

\author{
Xiangyun Shi, ${ }^{1}$ Yang Kuang, ${ }^{2}$ Athena Makroglou, ${ }^{3}$ Sriprakash Mokshagundam, ${ }^{4}$ \\ and Jiaxu $\mathrm{Li}^{5, \mathrm{a})}$ \\ ${ }^{1}$ School of Mathematics and Statistics, Xinyang Normal University, Xinyang 464000, Henan, People's \\ Republic of China \\ ${ }^{2}$ School of Mathematical and Statistical Sciences, Arizona State University, Tempe, Arizona 85287-1804, USA \\ ${ }^{3}$ Department of Mathematics, University of Portsmouth, 1st Floor Lion Gate Bldg, Portsmouth PO1 $3 \mathrm{HE}$, \\ United Kingdom \\ ${ }^{4}$ Department of Medicine, School of Medicine, University of Louisville, Louisville, Kentucky 40292, USA \\ ${ }^{5}$ Department of Mathematics, University of Louisville, Louisville, Kentucky 40292, USA
}

(Received 15 May 2017; accepted 8 September 2017; published online 9 November 2017)

\begin{abstract}
Type 2 diabetes mellitus (T2DM) has become prevalent pandemic disease in view of the modern life style. Both diabetic population and health expenses grow rapidly according to American Diabetes Association. Detecting the potential onset of T2DM is an essential focal point in the research of diabetes mellitus. The intravenous glucose tolerance test (IVGTT) is an effective protocol to determine the insulin sensitivity, glucose effectiveness, and pancreatic $\beta$-cell functionality, through the analysis and parameter estimation of a proper differential equation model. Delay differential equations have been used to study the complex physiological phenomena including the glucose and insulin regulations. In this paper, we propose a novel approach to model the time delay in IVGTT modeling. This novel approach uses two parameters to simulate not only both discrete time delay and distributed time delay in the past interval, but also the time delay distributed in a past sub-interval. Normally, larger time delay, either a discrete or a distributed delay, will destabilize the system. However, we find that time delay over a sub-interval might not. We present analytically some basic model properties, which are desirable biologically and mathematically. We show that this relatively simple model provides good fit to fluctuating patient data sets and reveals some intriguing dynamics. Moreover, our numerical simulation results indicate that our model may remove the defect in well known Minimal Model, which often overestimates the glucose effectiveness index. Published by AIP Publishing.

https://doi.org/10.1063/1.5008384
\end{abstract}

Delay differential equations (DDEs) have been frequently used to study complex dynamics observed in nature. More recently, they are used to understand intriguing physiological phenomena such as those expressed by glucose and insulin interaction. We propose a simple set of delay differential equations to model an intravenous glucose tolerance test. This model uses two parameters to simulate not only both discrete time delay and distributed time delay in the past interval, but also the time delay distributed in a past sub-interval. We show that this relatively simple model provides good fit to fluctuating patient data sets and reveals some intriguing dynamics. Most importantly, our model may remove the defect in the well known Minimal Model (MM) which often overestimates the glucose effectiveness (GE) index.

\section{INTRODUCTION}

Diabetes Mellitus continues to be a leading public health concern, contributing to an overwhelming number of debilitating health ailments, pre-mature death, and billions of dollars ( $\$ 245$ billion in the US in 2012) in medical care costs.

\footnotetext{
${ }^{\text {a) }}$ Author to whom correspondence should be addressed: jiaxu.li@louisville. edu
}

Approximately 29.1 million or $12.3 \%$ of American adults age 20 or older have diabetes. ${ }^{1}$ Worldwide, there are an estimated 422 million individuals with diabetes mellitus. Additionally, 79 million individuals have prediabetes, a condition that progresses to Type 2 diabetes mellitus (T2DM) at higher than normal rate. The pathogenesis of type 2 diabetes is complex and is mainly related to a progressive increase in insulin resistance (IR) and decline in insulin secretion, while other factors including changes in incretin hormones and renal handling of glucose excretion also play a role. ${ }^{2}$ Defects in insulin resistance and insulin secretion are seen early in the course of disease, in fact in the prediabetes phase. ${ }^{3}$ Understanding the mechanisms of insulin resistance and defective insulin secretion could provide an opportunity to develop strategies to prevent and treat diabetes.

Insulin is secreted from pancreas in a pulsatile manner under basal conditions with a frequency of 5 to $15 \mathrm{~min}$. Such regular oscillatory secretion is impaired in type 2 diabetes mellitus (T2DM). ${ }^{4}$ Defects in pulsatile insulin secretion have been demonstrated in early T2DM and in the relatives of subjects with T2DM. ${ }^{5}$ Insulin response to a glucose load sometimes occurs in two phases-a rapid first phase with dramatic increase in insulin levels, followed by a smaller but prolonged second phase that can form a second peak in insulin levels. Loss of first phase insulin secretion occurs early in 
the course of prediabetes and T2DM. ${ }^{3}$ Legacy work by O'Rahilly et al. ${ }^{6}$ showed that a significant peak at 13 to 14 min exists in the insulin dynamics of the non-diabetic people, but it is lacking in type 2 diabetic individuals and the relatives of T2DM. Thus, it is concluded that the lack of expected oscillatory insulin secretion in the first degree relatives of type 2 diabetics is believed to be an early diagnostics in the progression of type 2 diabetes. ${ }^{6}$ This peak at 13 to 14 min mark could be caused by the time lag from the insulin secretion to when the insulin is transported to interstitial space where insulin helps cells to uptake glucose. Furthermore, reduced glucose effectiveness is also an early change in T2DM and has been noted to be reduced in nondiabetic relatives of individuals with T2DM. ${ }^{7}$

A key methodological aspect of evaluating insulin resistance and insulin secretion is the development of methods to accurately measure these parameters reliably in a wide variety of physiological situations. The Frequently Sampled Intravenous glucose Tolerance test (fsIVGTT) has been widely used as tool to measure insulin sensitivity (IS), insulin secretion, and glucose effectiveness (GE) ${ }^{8}$ In fsIVGTT, after an overnight fasting, the subject is given a bolus of glucose $(300 \mathrm{~g} / \mathrm{kg})$ through intravenous, followed by sampling plasma glucose and serum insulin at time marks $2^{\prime}, 4^{\prime}, 6^{\prime}, 8^{\prime}$, $10^{\prime}, 12^{\prime}, 14^{\prime}, 18^{\prime}, 21^{\prime}, 24^{\prime}, 30^{\prime}, 35^{\prime}, 40^{\prime}, 45^{\prime}, 50^{\prime}, 60^{\prime}, 70^{\prime}, 80^{\prime}$, $90^{\prime}, 100^{\prime}, 120^{\prime}, 140^{\prime}, 160^{\prime}$, and $180^{\prime}$. The sampled data are often analyzed by using a mathematical model, and rich physiological information is thus revealed, including insulin sensitivity and glucose effectiveness. The values of the insulin sensitivity index $S_{i}$ (in the order of $10^{-5}$ or $10^{-4}$ ) and the glucose effectiveness index $S_{g}$ (in the order of $10^{-2}$ and $10^{-3}$ ) are calculated by the expressions of the parameters of the mathematical model. The most widely used model is the Minimal Model (MM) by Bergman et al. ${ }^{9,10}$ The insulin sensitivity index $\left(S_{i}\right)$ is closely related to the IS determined by the hyperinsulinemic euglycemic glucose clamp test. ${ }^{11} \mathrm{~A}$ commercial software, Penn Millennium MINMOD, ${ }^{12}$ was developed based on MM. ${ }^{13,14}$ However, it has been noticed that MM overestimates glucose effectiveness. ${ }^{15,16}$

The MM is a three dimensional ordinary differential equation (ODE) system including glucose concentration in plasma and insulin concentration in interstitial space as state variables, while insulin concentration in plasma as an input to the interstitial space. The split of the two compartments can be thought of implicitly simulating the time delay of insulin secretion stimulated by the elevated glucose level. Since then, many IVGTT and related models have been proposed in the forms of delay differential equation (DDE) systems in a single compartment incorporating the time delay explicitly. ${ }^{17-22}$ De Gaetano and Arino ${ }^{18}$ in 2000 introduced the following aggregated DDE model with a distributed time delay over the past interval

$$
\left\{\begin{array}{l}
G^{\prime}(t)=b_{7}-b_{1} G-b_{4} G I \\
I^{\prime}(t)=\frac{b_{6}}{b_{5}} \int_{t-b_{5}}^{t} G(s) d s-b_{2} I
\end{array}\right.
$$

with the initial conditions $G(0)=G_{b}+b_{0}, I(0)=I_{b}+b_{3} b_{0}$ and $G(t)=G_{b}, t \in\left[-b_{5}, 0\right)$, the constant parameters $b_{7}, b_{1}, b_{4}, b_{6}, b_{5}$ and $b_{2}$ stand for hepatic glucose production, glucose effectiveness, insulin sensitivity, maximal insulin secretion stimulated by glucose, time delay, and insulin degradation rate, respectively. In 2001, Li et al. ${ }^{23}$ generalized the Model (1.1) to the following form:

$$
\left\{\begin{array}{l}
G^{\prime}(t)=b_{7}-f(G(t))-g(G(t), I(t)), \\
I^{\prime}(t)=q\left(L\left(G_{t}\right)\right)-p(I(t)),
\end{array}\right.
$$

where $f(G), g(G, I)$ and $p(I)$ stand for insulin independent glucose uptake, insulin dependent glucose utilization and insulin degradation, respectively. $q\left(L\left(G_{t}\right)\right)$ is the insulin secretion stimulated by glucose with discrete time delay $L\left(G_{t}\right)=G\left(t-b_{5}\right)$ or distributed time delay $L\left(G_{t}\right)=\frac{1}{b_{5}}$ $\int_{-b_{5}}^{0} G(t+\theta) d \theta$. The readers are referred to Ref. 23 for the shapes of the function $f, g, p$ and $q$ and relevant details. It is demonstrated in Ref. 23 that the Hopf bifurcation exists for both types of time delays, distributed in the whole past interval $\left[-b_{5}, 0\right]$ and discretely at a single point back $b_{5}$ minutes. Later, De Gaetano and his colleagues also generalized the Model (1.1) to a family of DDE models in $2007^{22}$ in which general delays in both insulin action on tissue glucose uptake and in the glucose action on pancreatic insulin secretion are considered. On the other hand, De Gaetano and his colleagues claimed that a simplified single discrete time delay model ${ }^{24}$ is good enough to test the insulin sensitivity, and they discussed further the advantage of the simplified model. ${ }^{25}$ In 2012, Li et al. ${ }^{26}$ discussed the range of time delay and provided easy-to-check conditions for the globally asymptotic stability of the equilibrium point for the IVGTT model with a discrete time delay through Liapunov function approach.

Time delay of insulin secretion stimulated by increasing glucose may well be distributed in a past time sub-interval $\left[t-\tau_{2}, t-\tau_{1}\right]$, instead of concentrated at a single time of the past (a discrete delay), or evenly distributed in a whole past interval $[t-\tau, t]$. In this paper, we introduce a novel and generic approach to modeling such delayed effect as follows:

$$
\left\{\begin{array}{l}
G^{\prime}(t)=b-S_{g} G-S_{i} G I \\
I^{\prime}(t)=\sigma f\left(L\left(G_{t}\right)\right)-d_{i} I
\end{array}\right.
$$

with initial conditions $G(t)=\phi(t), t \in[-\tau-\epsilon, 0]$, where $b, S_{g}, S_{i}, \sigma$, and $d_{i}>0$ are parameters for the hepatic glucose production, glucose effectiveness, insulin sensitivity, maximal rate of insulin secretion stimulated by glucose and the insulin degradation rate, respectively. Two parameters $\tau>0$ and $0<\epsilon \leq \tau$ together represent the time delay of insulin secretion as below

$$
L\left(G_{t}\right)=\frac{1}{2 \epsilon} \int_{-\tau-\epsilon}^{-\tau+\epsilon} G(t+\theta) d \theta
$$

and

$$
f(x)=\frac{x^{n}}{\alpha^{n}+x^{n}} \quad \text { for } x \geq 0,
$$

is a sigmoidal function with $n \geq 2$ and the half saturation value $\alpha>0 . f(x)$ depicts the phenomenon that when insulin 
secretion is low when glucose level is low, but increases quickly when glucose level is elevated to some threshold level. ${ }^{27}$ The time delay in (1.4) is distributed in the interval $[-\tau-\epsilon,-\tau+\epsilon]$. In other words, the secretion rate of insulin at current time $t>0$ is related to the glucose level in the past sub-interval $[t-\tau-\epsilon, t-\tau+\epsilon]$. For the sake of convenience, we call average time delay $\tau$ the center of delay and $\epsilon$ the radius of the delay interval.

It is easy to see that $L\left(G_{t}\right)=\frac{1}{2 \tau} \int_{-2 \tau}^{0} G(t+\theta) d \theta$ when $\epsilon=\tau$. It takes the same form as $b_{5}=2 \tau$ in the model (2.3) in Ref. 23 and Eq. (5) in Ref. 18.

When $\epsilon \rightarrow 0, L\left(G_{t}\right)=G(t-\tau)$, it reduces to the discrete delay $L\left(G_{t}\right)=G(t-\tau)$ in the model (2.2) in Ref. 23 . Then (1.3) can be rewritten as

$$
\left\{\begin{array}{l}
G^{\prime}(t)=b-S_{g} G-S_{i} G I \\
I^{\prime}(t)=\sigma f(G(t-\tau))-d_{i} I
\end{array}\right.
$$

where $G(t)=\phi(t), t \in[-\tau, 0]$. According to Lebesgue's Dominated Convergence Theorem, we can show that the solution $\left(G_{\epsilon}(t), I_{\epsilon}(t)\right)$ of (1.3) tends to the solution $(G(t), I(t))$ of (1.6) as $\epsilon \rightarrow 0$.

The initial conditions of Model (1.3) are

$$
\varphi(t)=\left\{\begin{array}{l}
G_{b}, \text { for } t \in[-\tau-\epsilon,-\delta], \\
G_{0}+\delta^{-1}\left(G_{0}-G_{b}\right) t, \text { for } t \in[-\delta, 0],
\end{array}\right.
$$

while $I(0)=I_{b}$. Here, $G_{b}$ and $I_{b}$ are the basal levels of the blood glucose and insulin concentration levels, $t=0$ is the ending time of the bolus glucose infusion, and $0<\delta<\tau$ is the total time needed for the bolus glucose infusion and the time for glucose and insulin to increase from basal levels to the first peak levels. Usually, it takes 2 min for glucose infusion.

We organize this paper as follows. In Sec. II, the positivity and uniform boundedness of the solutions of the model is established. In fact, we also show solutions are eventually uniformly bounded away from zero. In Sec. III, we provide some sufficient conditions that ensure the unique positive equilibrium is asymptotically or globally asymptotically stable. In Sec. IV, we investigate the existence of the Hopf bifurcation when $\tau$ or $\epsilon$ varies and when both vary. In Sec. V, we fit our model with clinical data sets and demonstrate some of the interesting phenomena revealed by the new model. A brief discussion on the implications of our results are presented in the last section.

\section{PRELIMINARIES}

In this section, we present some basic results, such as the positive invariance of system (1.3), the existence of equilibrium, the boundedness of solutions and the persistence of the system.

Proposition 2.1. The solutions $(G(t), I(t))$ of system (1.3) are positive and bounded.

Proof. Let $(G(t), I(t))$ be a solution of System (1.3). If there exists a $t_{0}>0$ such that $G\left(t_{0}\right)=0$ but $G(t)>0$ for $t \in\left[0, t_{0}\right]$, then $G^{\prime}\left(t_{0}\right) \leq 0$. But the first equation of (1.3) implies that $G^{\prime}\left(t_{0}\right)=b>0$. This shows that $G(t)>0$ for all $t$ in the interval of its existence. Similarly, if there exists a $\bar{t}_{0}>0$ such that $I\left(\bar{t}_{0}\right)=0$ but $I(t)>0$ for $t \in\left[0, \bar{t}_{0}\right]$, then from the second equation of (1.3), $I^{\prime}\left(\bar{t}_{0}\right)=\sigma f\left(L\left(G\left(\bar{t}_{0}\right)\right)\right)$ $-d_{2} I\left(\bar{t}_{0}\right)=\sigma f\left(L\left(G\left(\bar{t}_{0}\right)\right)\right.$. Since $G_{\bar{t}_{0}}(\theta)=G\left(\bar{t}_{0}+\theta\right)>0$ for $\theta \in[-\tau-\epsilon, 0]$, then $f\left(L\left(G\left(\bar{t}_{0}\right)\right)\right)>0$ by the definition of the function $f$. Thus, $I^{\prime}\left(\bar{t}_{0}\right)>0$, which shows that $I(t)>0$ for all $t$ in the interval of its existence. As for the boundedness of $G(t)$, by the first equation of (1.3)

$$
G^{\prime}(t) \leq b-S_{g} G
$$

Thus, $G(t)$ is bounded by $G_{M}:=\max \left\{\phi(t), b / S_{g}\right\}$. Then, by the second equation of (1.3) we can obtain

$$
I^{\prime}(t) \leq \sigma f\left(G_{M}\right)-d_{i} I .
$$

Thus, $I(t)$ is bounded by $I_{M}:=\max \left\{I(0), \sigma f\left(G_{M}\right) / d_{i}\right\}$. The boundedness statement implies that the solution exists for all $t>0$.

Proposition 2.2. System (1.3) is persistent, that is, solutions of (1.3) are ultimately bounded from above and from below by a positive lower bound.

Proof. From the proof of Proposition 2.1, we know that

$$
G(t)<G_{M} \text { for all } t>0
$$

and

$$
I(t)<I_{M} \text { for all } t>0 .
$$

Thus, we only need to find the solutions' positive bound from below.

From the first equation of (1.3) and (2.2), we have

$$
G^{\prime}(t) \geq b-S_{g} G-S_{i} G I_{M}
$$

Therefore,

$$
\begin{gathered}
G(t) \geq\left(G(0)-\frac{b}{S_{g}+S_{i} I_{M}}\right) e^{-\left(S_{g}+S_{i} I_{M}\right) t} \\
+\frac{b}{S_{g}+S_{i} I_{M}} \quad \text { for all } t>0
\end{gathered}
$$

which implies that

$$
G(t) \geq \frac{b}{S_{g}+S_{i} I_{M}}-\eta_{1}:=G_{m}>0 \text { for some } \eta>0 .
$$

Then, from the second equation of (1.3), we can derive that

$$
I^{\prime}(t) \geq \sigma f\left(G_{m}\right)-d_{i} I .
$$

Hence

$$
I(t) \geq\left(I(0)-\frac{\sigma f\left(G_{m}\right)}{d_{i}}\right) e^{-d_{i} t}+\frac{\sigma f\left(G_{m}\right)}{d_{i}} \text { for } t>0 .
$$

Then, for some $\eta>0$

$$
I(t)>\frac{\sigma f\left(G_{m}\right)}{d_{i}}-\eta:=I_{m} .
$$

From above, we conclude that the solutions of (1.3) are eventually bounded from above and away from zero. 


\section{STABILITY OF THE EQUILIBRIUM}

In this section, we will discuss the local and global stability of the positive equilibrium $\left(G^{*}, I^{*}\right)$ of system (1.3), respectively.

Consider the algebraic equations defining any equilibrium point $\left(G^{*}, I^{*}\right)$

$$
\left\{\begin{array}{l}
b-S_{g} G^{*}-S_{i} G^{*} I^{*}=0 \\
\sigma f\left(G^{*}\right)-d_{i} I^{*}=0
\end{array}\right.
$$

Clearly

$$
I^{*}=\frac{\sigma f\left(G^{*}\right)}{d_{i}}
$$

and

$$
b-S_{g} G^{*}-S_{i} G^{*} \frac{\sigma f\left(G^{*}\right)}{d_{i}}=0 .
$$

Let

$$
F(G)=b-S_{g} G-S_{i} G \frac{\sigma f(G)}{d_{i}} \quad \text { for all } G \geq 0 .
$$

Then, $F(0)=b>0$ and

$F(+\infty)=\lim _{G \rightarrow+\infty} F(G)=\lim _{G \rightarrow+\infty}\left(b-S_{g} G-S_{i} G \frac{\sigma f(G)}{d_{i}}\right)=-\infty$.

Furthermore

$$
\frac{d F(G)}{d G}=-S_{g}-\frac{S_{i} \sigma}{d_{i}} f(G)-\frac{S_{i} \sigma}{d_{i}} G f^{\prime}(G)
$$

Since $f(G)>0$ for $G>0, f^{\prime}(G)>0$ for $G>0$. Thus, $F(\cdot)$ is a decreasing function for the arguments and it may hence have a unique positive $\operatorname{root}\left(G^{*}, I^{*}\right)$. That is, the Model (1.3) has a unique equilibrium point $\left(G^{*}, I^{*}\right)$.

In IVGTT, the blood glucose and insulin concentration levels return to their basal level after about three hours. So, we assume that $G^{*}=G_{b}$ and $I^{*}=I_{b}$. Then, we have

$$
\left\{\begin{array}{l}
b-S_{g} G_{b}-S_{i} G_{b} I_{b}=0 \\
\sigma f\left(G_{b}\right)-d_{i} I_{b}=0
\end{array}\right.
$$

Thus

$$
d_{i}=\frac{\sigma f\left(G_{b}\right)}{I_{b}} \text { and } b=S_{g} G_{b}-S_{i} G_{b} I_{b}
$$

and therefore, the number of parameters of the Model (1.3) is reduced to 7 .

Applying the same method of Theorem 3.1 in Ref. 23, the following straight forward result is proved, which is consistent with the conclusion $\left(A_{4}\right)$ for the discrete delay model in Ref. 26 on the global stability of the positive steady state.

Theorem 3.1. If $n \leq 1$, then the unique equilibrium point $\left(G^{*}, I^{*}\right)$ of $(1.3)$ is globally asymptotically stable.

The above result on stability does not depend on the value of the delay. In clinical test, the insulin release is abrupt stimulated by the bolus glucose infusion into intravenous, which implies that $n>1$ to model the abrupt insulin release. We now turn to consider the case for $n>1$.

Consider the model (1.3). Let $G_{1}(t)=G(t)-G^{*}$, $I_{1}(t)=I(t)-I^{*}$, then model (1.3) is transformed to

$$
\left\{\begin{array}{l}
G_{1}^{\prime}(t)=b-S_{g}\left(G_{1}(t)+G^{*}\right)-S_{i}\left(G_{1}(t)+G^{*}\right)\left(I_{1}(t)+I^{*}\right), \\
I_{1}^{\prime}(t)=\sigma f\left(L\left(\left(G_{1}\right)_{t}\right)+G^{*}\right)-d_{i}\left(I_{1}(t)+I^{*}\right) .
\end{array}\right.
$$

The unique equilibrium point $\left(G^{*}, I^{*}\right)$ is mapped to $(0,0)$ for (3.2). The linearized system of (3.2) about its equilibrium point is given by

$$
\left\{\begin{array}{l}
G_{2}^{\prime}(t)=\left(-S_{g}-S_{i} I^{*}\right) G_{2}(t)-S_{i} G^{*} I_{2}(t) \\
I_{2}^{\prime}(t)=-d_{i} I_{2}(t)+\sigma f^{\prime}\left(G^{*}\right) L\left(\left(G_{2}\right)_{t}\right),
\end{array}\right.
$$

and the corresponding characteristic equation of (3.3) is

$$
\left|\begin{array}{cc}
\lambda+S_{g}+S_{i} I^{*} & S_{i} G^{*} \\
-\frac{\sigma f^{\prime}\left(G^{*}\right)}{2 \epsilon} \int_{-\tau-\epsilon}^{-\tau+\epsilon} e^{\lambda \theta} d \theta & \lambda+d_{i}
\end{array}\right|=0,
$$

that is,

$$
\begin{gathered}
\lambda^{2}+\left(S_{g}+S_{i} I^{*}+d_{i}\right) \lambda+d_{i}\left(S_{i} I^{*}+S_{g}\right) \\
+\frac{S_{i} G^{*} \sigma f^{\prime}\left(G^{*}\right)}{2 \epsilon} \int_{-\tau-\epsilon}^{-\tau+\epsilon} e^{\lambda \theta} d \theta=0 .
\end{gathered}
$$

Denote

$a=S_{g}+S_{i} I^{*}+d_{i}, c=d_{i}\left(S_{i} I^{*}+S_{g}\right)$ and $h=S_{i} G^{*} \sigma f^{\prime}\left(G^{*}\right)$.

Clearly $a^{2}-2 c>0$, which is applied in Sec. IV A, and we can rewrite (3.4) as

$$
\lambda^{2}+a \lambda+c+\frac{h}{2 \epsilon} \int_{-\tau-\epsilon}^{-\tau+\epsilon} e^{\lambda \theta} d \theta=0 .
$$

When there is no delay, that is $\tau=0$ and $\epsilon=0$, the characteristic equation becomes

$$
\lambda^{2}+a \lambda+c=0
$$

and the real parts of the eigenvalues are negative, which implies that the trivial solution is globally asymptotically stable.

If the trivial solution of (3.3) is unstable for some $\tau>0$ and $\epsilon>0$, there must exist $\omega>0$ such that $\lambda=i \omega$ is a solution of (3.6), i.e.,

$$
(i \omega)^{2}+a \omega i+c+\frac{h}{2 \epsilon} \int_{-\tau-\epsilon}^{-\tau+\epsilon}(\cos (\omega \theta)+i \sin (\omega \theta)) d \theta=0,
$$

that is,

$$
-\omega^{2}+a \omega i+c+\frac{h}{2 \epsilon} \int_{-\tau-\epsilon}^{-\tau+\epsilon} \cos (\omega \theta) d \theta+i \frac{h}{2 \epsilon} \int_{-\tau-\epsilon}^{-\tau+\epsilon} \sin (\omega \theta) d \theta=0 .
$$

Thus, we have 


$$
-\omega^{2}+c+\frac{h}{2 \epsilon} \int_{-\tau-\epsilon}^{-\tau+\epsilon} \cos \omega \theta d \theta=0
$$

and

$$
a \omega+\frac{h}{2 \epsilon} \int_{-\tau-\epsilon}^{-\tau+\epsilon} \sin \omega \theta d \theta=0,
$$

which leads to

$$
a=h \tau\left(\frac{\sin \omega \epsilon}{\omega \epsilon}\right)\left(\frac{\sin \omega \tau}{\omega \tau}\right) \leq h \tau .
$$

Thus, we have proved the following theorem.

Theorem 3.2. If the delay center $\tau<\frac{a}{h}$, then the trivial solution of the linear system of the model (3.3) is globally asymptotically stable.

Consequently, we have

Theorem 3.3. If the delay center

$$
\tau<\tau_{b}:=\frac{a}{h}=\left(S_{g}+S_{i} I_{b}+d_{i}\right) /\left(\sigma S_{i} G_{b} f^{\prime}\left(G^{*}\right)\right),
$$

then the positive equilibrium $\left(G^{*}, I^{*}\right)$ of (1.3) is locally asymptotically stable.

Remark 3.4. From Theorem 3.1 and Theorem 3.3, we find that when the parameters in the Model (1.3) are fixed, the shape of the function $f(x)=\frac{x^{n}}{\alpha^{n}+x^{n}}$ will affect the stability of the Model (1.3). If $n \leq 1, f$ is in the shape of the Michaelis-Menten kinetics. From Theorem 3.1, the model is globally asymptotically stable independent of the time delay. If $n>1, f(x)$ is in the sigmoidal shape. Notice that the inflection point of $f$ is at $G_{r}=\left(\frac{n-1}{n+1}\right)^{\frac{1}{n}} \alpha<\alpha$ as $n>1$. The slope of $f$ attains its maximal value at $G_{r}$. Theorem 3.3 provides a bound $\tau_{b}$ for the delay center $\tau$ which depends on the slope of $f$ at the plasma glucose basal level. So if $G_{b}<\alpha$, then smaller $\alpha$ causes the bound $\tau_{b}$ smaller. When $G_{b}$ is near to $\alpha$, larger $n$ will cause larger $f^{\prime}\left(G_{b}\right)$ and therefore smaller $\tau_{b}$. Thus, the two parameters $n$ and $\alpha$ together along with large $\tau$ could destabilize the system (1.3). On the other hand, in the clinical setup of IVGTT, both glucose and insulin levels will return to their basal levels in about $3 \mathrm{~h}$. This suggests that Model (1.3) may not be destabilized with reasonable parameter values. In Sec. IV, we will study when the system becomes unstable.

\section{BIFURCATION ANALYSIS}

In this section, we seek Hopf bifurcations with respect to the parameters $\tau$ and $\epsilon$, respectively. We will show that for $\tau$ varying from small to large, the system (1.3) could become unstable, and however, for $\epsilon$ varying from small to large, the system (1.3) could change from unstable to stable.

\section{A. Hopf bifurcation with parameter $\tau$}

We first show that a local Hopf bifurcation takes place when the delay center $\tau$ varies.

Notice that (3.6) can be rewritten as

$$
F(\lambda)=\lambda^{2}+a \lambda+c+\frac{h}{2 \epsilon \lambda}\left(e^{(-\tau+\epsilon) \lambda}-e^{(-\tau-\epsilon) \lambda}\right)=0 .
$$

If as $\tau$ increases, the trivial solution becomes unstable, then there must be a $\tau^{*}>0$ such that when $\tau=\tau^{*}, F(i \omega)=0$ for some $\omega>0$. And thus

$$
(i \omega)^{2}+a i \omega+c+\frac{h}{2 \epsilon i \omega}\left(e^{(-\tau+\epsilon) i \omega}-e^{(-\tau-\epsilon) i \omega}\right)=0 .
$$

Equivalently,

$$
\begin{aligned}
& -\omega^{2}+a \omega i+c-\frac{h}{2 \epsilon \omega}[(-2 \sin (-\omega \tau) \sin (\omega \epsilon)) i \\
& +2 \cos (-\omega \tau) \sin (-\omega \epsilon)]=0 .
\end{aligned}
$$

Hence,

$$
\left\{\begin{array}{l}
\cos (\omega \tau) \sin (\omega \epsilon)=\frac{\left(\omega^{2}-c\right) \epsilon \omega}{h}, \\
\sin (\omega \tau) \sin (\omega \epsilon)=\frac{a \epsilon \omega^{2}}{h}
\end{array}\right.
$$

and

$$
\left\{\begin{array}{l}
\omega^{4}+\left(a^{2}-2 c\right) \omega^{2}+c^{2}=\frac{h^{2}}{\epsilon^{2} \omega^{2}} \sin ^{2}(\omega \epsilon), \\
\tau=\frac{1}{\omega} \cot ^{-1} \frac{\omega^{2}-c}{a \omega} .
\end{array}\right.
$$

Let

$$
g(\omega)=\omega^{4}+\left(a^{2}-2 c\right) \omega^{2}+c^{2}-\frac{h^{2}}{\epsilon^{2} \omega^{2}} \sin ^{2} \omega \epsilon .
$$

From (4.4), it is easy to find that

$$
g\left(0^{+}\right)=c^{2}-h^{2},
$$

and

$$
g\left(\frac{\pi}{\epsilon}\right)=\left(\frac{\pi}{\epsilon}\right)^{4}+\left(a^{2}-2 c\right)\left(\frac{\pi}{\epsilon}\right)^{2}+c^{2}>0 .
$$

If $c<h$, then $g\left(0^{+}\right)<0$. Therefore, there exists at least one root $\hat{\omega} \in\left(0, \frac{\pi}{\epsilon}\right)$ such that $g(\hat{\omega})=0$.

Notice that $a^{2}-2 c>0$ and $\sin x-x \cos x>0$ for $0<x<\pi$. Thus

$$
\begin{aligned}
g^{\prime}(\omega)= & 4 \omega^{3}+2\left(a^{2}-2 c\right) \omega-\frac{2 h^{2}}{\epsilon \omega^{2}} \cos (\omega \epsilon) \sin (\omega \epsilon) \\
& +\frac{2 h^{2}}{\epsilon^{2} \omega^{3}} \sin ^{2}(\omega \epsilon) \\
= & 4 \omega^{3}+2\left(a^{2}-2 c\right) \omega+\frac{2 h^{2} \sin \epsilon \omega}{\epsilon \omega^{2}} \\
& \times\left(\frac{\sin \epsilon \omega-\epsilon \omega \cos \epsilon \omega}{\epsilon \omega}\right)>0,
\end{aligned}
$$

for $0<\omega<\frac{\pi}{\epsilon}$. So we obtain the following lemma.

Lemma 4.1. If $c<h$, then the function $g(\omega)$ in (4.4) has a unique zero $\hat{\omega} \in\left(0, \frac{\pi}{\epsilon}\right)$.

Lemma 4.1 implies that there exists a $\hat{\omega}>0$ such that $F(i \hat{\omega})=0$, which leads to 
Theorem 4.2. If $c<h$, then the system (1.3) undergoes a Hopf bifurcation at

$$
\hat{\tau}:=\frac{1}{\hat{\omega}} \cot ^{-1}\left(\frac{\hat{\omega}^{2}-c}{a \hat{\omega}}\right), \quad \text { for } 0<\hat{\omega}<\frac{\pi}{\epsilon},
$$

when $\tau>0$ increases.

The proof is given in Appendix A.

Remark 4.3. From Theorem 4.2, it is easy to find that model (1.3) undergoes a Hopf bifurcation related not only to the value of $\tau$, but also to the value of $\epsilon$. We shall demonstrate numerical investigation in Sec. V.

Next, we shall compare the delay lengths of the Hopf bifurcation in the three different cases: discrete delay, distributed delay in the past whole interval, and the distributed delay in the past sub-interval. In fact, the three type delay models can be considered in the following three cases: (A) $\epsilon \rightarrow 0$, (B) $\epsilon=\tau$, and (C) $0<\epsilon<\tau$.

In case (C), let $\hat{\tau}$ and $\hat{\omega}$ satisfy (4.6). Now, we compare the bifurcation value $\hat{\tau}$ with the bifurcation values in other two cases.

Case (A). For $\epsilon \rightarrow 0^{+}$, the characteristic equation of (3.3) becomes

$$
\lambda^{2}+a \lambda+c+h e^{-\tau \lambda}=0 .
$$

If $i \omega_{1}$ is the root of (4.7), similar to the above approach, we can obtain the following equation corresponding to the first equation of (4.3):

$$
\omega_{1}^{4}+\left(a^{2}-2 c\right) \omega_{1}^{2}+c^{2}=h^{2} .
$$

Then, we can find that if $c<h$, Eq. (4.8) has a positive root

$$
\omega_{1}=\sqrt{\frac{-\left(a^{2}-2 c\right)+\sqrt{\left(a^{2}-2 c\right)^{2}-4\left(c^{2}-h^{2}\right)}}{2} .}
$$

In this case, $\tau=\tau_{1}=\frac{1}{\omega_{1}} \cot ^{-1}\left(\frac{\omega_{1}^{2}-c}{a \omega_{1}}\right)$. Using the same method in the proof of Theorem 4.2, we can also obtain the result: For $\epsilon \rightarrow 0$, if $c<h$ holds, then (1.3) undergoes a Hopf bifurcation when $\tau=\tau_{1}=\frac{1}{\omega_{1}} \cot ^{-1}\left(\frac{\omega_{1}^{2}-c}{a \omega_{1}}\right)$, and $\omega_{1}=$ $\sqrt{\frac{-\left(a^{2}-2 c\right)+\sqrt{\left(a^{2}-2 c\right)^{2}-4\left(c^{2}-h^{2}\right)}}{2}}$. Comparing the first equation of (4.3) and (4.8), we can easily get $\omega_{1}>\hat{\omega}$. That is, for the system (1.3), the bifurcation value in the case of the discrete time delay is smaller than that in the case of the distributed time delay. In other words, when the delay parameter $\tau$ increases, the system with discrete time delay is sooner to be destabilized.

Case (B). For $\epsilon=\tau$, the first equation of (4.3) can be rewritten as

$$
\omega_{2}^{4}+\left(a^{2}-2 c\right) \omega_{2}^{2}+c^{2}=\frac{h^{2}}{\tau^{2} \omega_{2}^{2}} \sin ^{2} \tau \omega_{2} .
$$

If a positive root $\omega_{2}$ of (4.9) exists under some conditions, then model (1.3) may undergo a bifurcation at $\tau_{2}=\frac{1}{\omega_{2}} \cot ^{-1} \frac{\omega_{2}^{2}-c}{a \omega_{2}}$. In the case $(\mathrm{C}), \epsilon<\tau$, so we can compare the value of the right hand side of the first equation (4.3) with the right hand side of (4.9). Since $\frac{\sin (\epsilon \omega)}{\epsilon \omega}>\frac{\sin (\tau \omega)}{\tau \omega}$ for $0<\epsilon<\tau<\pi / \omega$, we know that the root of (4.9) $\omega_{2}<\hat{\omega}$. This possibly implies that the time delay distributed in a past sub-interval is more likely to destabilize a system than the time delay distributed in the whole past interval.

\section{B. Hopf bifurcation with parameter $\epsilon$}

In Sec. IV A, we have proved that the system can undergo a Hopf bifurcation when $\tau>0$ increases. In this subsection, we investigate the Hopf bifurcation of the system (1.3) when $\epsilon>0$ varies.

From Sec. IV A, we know that if $c<h$, then the strictly increasing function $g(\omega)$ in (4.4) has a unique zero $\hat{\omega}$ with $0<\hat{\omega}<\frac{\pi}{\epsilon}$. If further

$$
g\left(\frac{\pi}{2 \epsilon}\right)=\left(\frac{\pi}{2 \epsilon}\right)^{4}+\left(a^{2}-2 c\right)\left(\frac{\pi}{2 \epsilon}\right)^{2}+c^{2}-\frac{4 h^{2}}{\pi^{2}}<0,
$$

which is equivalent to

$$
\epsilon>\pi\left(2\left(-\left(a^{2}-2 c\right)+\sqrt{a^{4}-4 a^{2} c+16 h^{2} / \pi^{2}}\right)\right)^{-\frac{1}{2}},
$$

then the zero $\hat{\omega} \in\left(\frac{\pi}{2 \epsilon}, \frac{\pi}{\epsilon}\right)$. For this interval $\left(\frac{\pi}{2 \epsilon}, \frac{\pi}{\epsilon}\right)$, the characteristic equation (3.6) must have a unique pair of pure imaginary eigenvalues, which is dependent of $\tau$ and $\epsilon$.

From (4.3), we obtain that

$$
\frac{d \tau}{d \omega}=-\frac{1}{\omega^{2}} \cot ^{-1}\left(\frac{\omega^{2}-c}{a \omega}\right)-\frac{a \omega^{2}+a c}{\omega\left(1+\frac{\omega^{2}-c}{a \omega}\right)^{2}(a \omega)^{2}}<0,
$$

and

$$
\begin{aligned}
\frac{d \omega}{d \epsilon}= & \frac{-2 h^{2} \frac{\sin (\omega \epsilon)}{\epsilon^{2} \omega}\left(\frac{\sin (\epsilon \omega)}{\epsilon \omega}-\cos (\epsilon \omega)\right)}{4 \omega^{3}+2 \omega\left(a^{2}-c\right)+2 h^{2} \frac{\sin (\omega \epsilon)}{\epsilon \omega^{2}}\left(\frac{\sin (\epsilon \omega)}{\epsilon \omega}-\cos (\epsilon \omega)\right)} \\
& <0 \text { for } 0<\omega<\frac{\pi}{\epsilon} .
\end{aligned}
$$

Therefore,

$$
\frac{d \epsilon}{d \tau}>0 \text { for } 0<\omega<\frac{\pi}{\epsilon} .
$$

So, the relation between $\tau$ and $\epsilon$ determined by (4.3) is monotonic. Notice also $\omega \rightarrow 0^{+}$as $\tau \rightarrow \infty$ and $\omega \rightarrow \infty$ as $\tau \rightarrow 0^{+}$. Now, let $\tau^{*} \geq \hat{\tau}$ be fixed. Then, there exists a unique $\epsilon^{*}$ corresponding to $\tau^{*}$ that satisfies (4.3). We will show that Hopf bifurcation occurs at $\epsilon^{*}$ when $\epsilon>\epsilon^{*}$ decreases in the following theorem whose proof is given in Appendix B.

Theorem 4.4. Given fixed $\tau^{*}>\hat{\tau}$, let $\epsilon^{*}$ be determined by (4.3). Assume that (4.10) holds. Then, when $\epsilon>\epsilon^{*}$ decreases, the system (1.3) undergoes a Hopf bifurcation at $\epsilon^{*}>0$, namely, the unique equilibrium point $\left(G^{*}, I^{*}\right)$ losses 
its stability and the system has a periodic solution, where $\tau^{*}$ and $\epsilon^{*}$ satisfy (4.3).

\section{Codimension-two bifurcation with parameters $\tau$ and $\epsilon$}

In Secs. IV A and IV B, we have shown the existence of Hopf bifurcation when the delay center $\tau$ increases or, for large $\tau$, when the delay interval radius $\epsilon$ decreases from large values. We now let both parameters $\tau$ and $\epsilon$ vary simultaneously and investigate the codimension-two bifurcation.

From (4.11), we see that $\tau$ is a decreasing function of $\omega$, while (4.12) implies that $\omega$ is a decreasing function of $\epsilon$. Therefore, by (4.13), the Hopf bifurcation value of $\tau$ increases when the Hopf bifurcation value $\epsilon$ increases, which ensures the existence of a monotonic curve $L$ formed by Hopf bifurcation points in the $(\tau, \epsilon)$-plane.

We take the data of subject no. 13 as an example for simulation. Our intensive simulations confirm the existence of the smooth curve $L$ dividing the $(\tau, \epsilon)$-plane into two regions $D_{s}$, in which the equilibrium point is stable, and $D_{u}$, in which a limit cycle exists. As expected by the analytical result given by (4.13), no degeneracy is found in our numerical investigations. Furthermore, (4.13) shows that $L$ is a monotonic curve. For clarity, we plot the curve $L$ only in the window $[180,280] \times[10,180]$ in the $(\tau, \epsilon)$-plane in Fig. 1 .

Remark 4.5. As aforementioned, under the clinical setup of IVGTT, the glucose and insulin concentrations will return to their basal levels, respectively. So, the steady state (at the basal levels) of the models that model the IVGTT should be stable with reasonable parameter values in physiological range. Figure 1 above and Figs. 4 and 5 in Sec. V show that Hopf bifurcation would only occur when the delay parameter values are large and out of physiological range.

\section{SIMULATIONS}

We have performed extensive numerical simulations using experimental data listed in Tables I and II in Ref. 26, which originated from Refs. 18 and 24, and the example data in Penn Millennium MINMOD, ${ }^{12}$ a commercial software developed according to MM. We compared the parameter

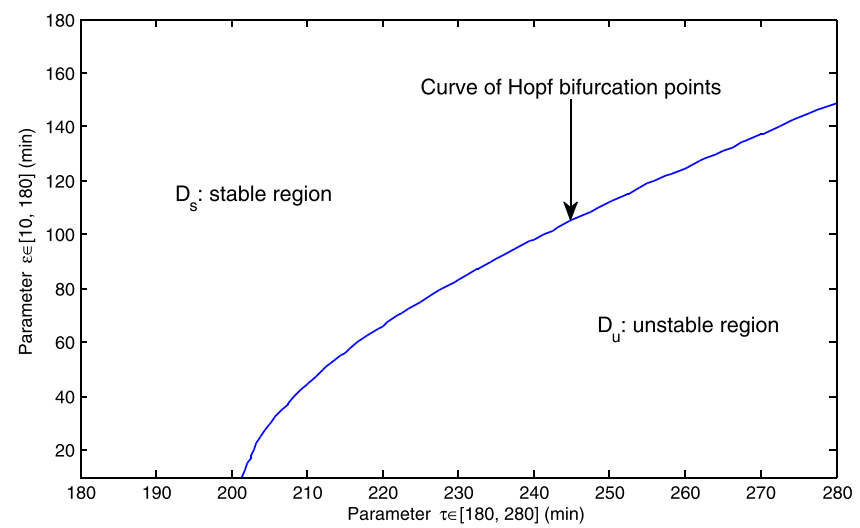

FIG. 1. Codimension-two bifurcation diagram for the subject no. 13 in Ref. 24 produced by Model (1.3). The bifurcation curve $L$ divides the $(\tau, \epsilon)$-plane into a stable region $D_{s}$ and an unstable region $D_{u}$.
TABLE I. Parameter values estimated by Model (1.3) for subject nos. 6, 7, 8, and 13 in Ref. 26.

\begin{tabular}{lcccc}
\hline \hline Subjects & No. 6 & No. 7 & No. 8 & No. 13 \\
\hline$S_{\mathrm{g}}$ & $1.6150 \times 10^{-02}$ & $2.1810 \times 10^{-02}$ & $1.5998 \times 10^{-02}$ & $3.5000 \times 10^{-02}$ \\
$S_{\mathrm{i}}$ & $5.4371 \times 10^{-05}$ & $2.0191 \times 10^{-04}$ & $3.5585 \times 10^{-05}$ & $2.3000 \times 10^{-05}$ \\
$b$ & $1.7431 \times 10^{+00}$ & $2.5178 \times 10^{+00}$ & $1.4085 \times 10^{+00}$ & $2.6379 \times 10^{+00}$ \\
$\sigma$ & $1.6388 \times 10^{+02}$ & $2.1861 \times 10^{+01}$ & $1.4109 \times 10^{+01}$ & $3.5340 \times 10^{-01}$ \\
$\alpha$ & $4.2050 \times 10^{+02}$ & $9.9628 \times 10^{+01}$ & $1.0000 \times 10^{+02}$ & $2.0000 \times 10^{+02}$ \\
$d_{\mathrm{i}}$ & $9.9250 \times 10^{-02}$ & $2.0946 \times 10^{-01}$ & $6.0000 \times 10^{-02}$ & $1.0500 \times 10^{-01}$ \\
$\tau$ & $8.2500 \times 10^{+00}$ & $1.2369 \times 10^{+01}$ & $1.4250 \times 10^{+01}$ & $1.8000 \times 10^{+01}$ \\
$\epsilon$ & $2.0000 \times 10^{+00}$ & $5.0000 \times 10^{+00}$ & $3.0000 \times 10^{+00}$ & $9.0000 \times 10^{+00}$ \\
$G_{\mathrm{M}}$ & $2.2547 \times 10^{+02}$ & $2.9937 \times 10^{+02}$ & $2.2642 \times 10^{+02}$ & $1.8340 \times 10^{+02}$ \\
$G_{\mathrm{m}}$ & $5.5929 \times 10^{+01}$ & $2.1908 \times 10^{+01}$ & $1.0448 \times 10^{+01}$ & $1.0307 \times 10^{+01}$ \\
$I_{\mathrm{M}}$ & $4.1321 \times 10^{+02}$ & $1.7945 \times 10^{+02}$ & $1.0314 \times 10^{+03}$ & $2.3100 \times 10^{+02}$ \\
$I_{\mathrm{m}}$ & $6.1622 \times 10^{+00}$ & $2.0800 \times 10^{-01}$ & $7.3000 \times 10^{-03}$ & $3.2100 \times 10^{-02}$ \\
\hline \hline
\end{tabular}

values estimated by our model to those in Refs. 18, 24, and 26 and Penn Millennium MINMOD. The profiles of our numerical simulations are shown in Figs. 2 and 3. The estimated parameters are listed in Tables I and II, respectively. Comparison of estimated parameter values $S_{g}$ and $S_{i}$ are shown in Table III. We noticed that the profiles obtained by Model (1.3) fit the data better in the following aspects.

(a) From Figs. 2 and 3, we observe that after a rapid insulin secretion, the insulin level may decline dramatically and rebound slightly;

(b) From Table III, we see that the glucose effectiveness index $S_{g}$ is smaller than that estimated by Penn Millennium MINMOD, ${ }^{12}$ which improves the known issue that MM overestimates the glucose effectiveness; ${ }^{15,16}$ and

(c) From Table III, we see that the values of $S_{g}$ in all simulations are in a realistic range, which may not be true for the results presented in Refs. 24 and 26.

We estimate the model parameters in a hybrid fashion. We initially determine the parameter values by the Least Square Method (LSM) and then empirically fine-tuned the values to fit the data. This allows us to search for better fittings that match well with the initial rapid insulin secretion, subsequent decline in insulin levels and the second peak

TABLE II. Parameter values estimated by Model (1.3) for Subject 27 in Ref. 26, MM Ex 1 and MM Ex 2 in Ref. 12.

\begin{tabular}{lccc}
\hline \hline Subjects & No. 27 & MM Ex 1 & MM Ex 2 \\
\hline$S_{\mathrm{g}}$ & $1.3630 \times 10^{-03}$ & $2.0600 \times 10^{-02}$ & $8.2897 \times 10^{-03}$ \\
$S_{\mathrm{i}}$ & $6.2029 \times 10^{-05}$ & $2.4930 \times 10^{-04}$ & $7.5621 \times 10^{-04}$ \\
$b$ & $3.5386 \times 10^{-01}$ & $1.8936 \times 10^{+00}$ & $1.5279 \times 10^{+00}$ \\
$\sigma$ & $1.7705 \times 10^{+01}$ & $1.0716 \times 10^{+03}$ & $5.7475 \times 10^{+00}$ \\
$\alpha$ & $9.7757 \times 10^{+01}$ & $7.0404 \times 10^{+02}$ & $1.0504 \times 10^{+02}$ \\
$d_{\mathrm{i}}$ & $9.9375 \times 10^{-02}$ & $1.6900 \times 10^{-01}$ & $1.9030 \times 10^{-01}$ \\
$\tau$ & $1.3914 \times 10^{+01}$ & $1.4066 \times 10^{+01}$ & $1.0066 \times 10^{+01}$ \\
$\epsilon$ & $3.0000 \times 10^{+00}$ & $5.0000 \times 10^{-01}$ & $5.0000 \times 10^{+00}$ \\
$G_{\mathrm{M}}$ & $3.4590 \times 10^{+02}$ & $3.1400 \times 10^{+02}$ & $3.5000 \times 10^{+02}$ \\
$G_{\mathrm{m}}$ & $3.7389 \times 10^{+00}$ & $1.2628 \times 10^{+01}$ & $5.1966 \times 10^{+00}$ \\
$I_{\mathrm{M}}$ & $1.0360 \times 10^{+03}$ & $2.2900 \times 10^{+02}$ & $2.3869 \times 10^{+02}$ \\
$I_{\mathrm{m}}$ & $2.8365 \times 10^{-011}$ & $1.6000 \times 10^{-04}$ & $7.8862 \times 10^{-04}$ \\
\hline \hline
\end{tabular}



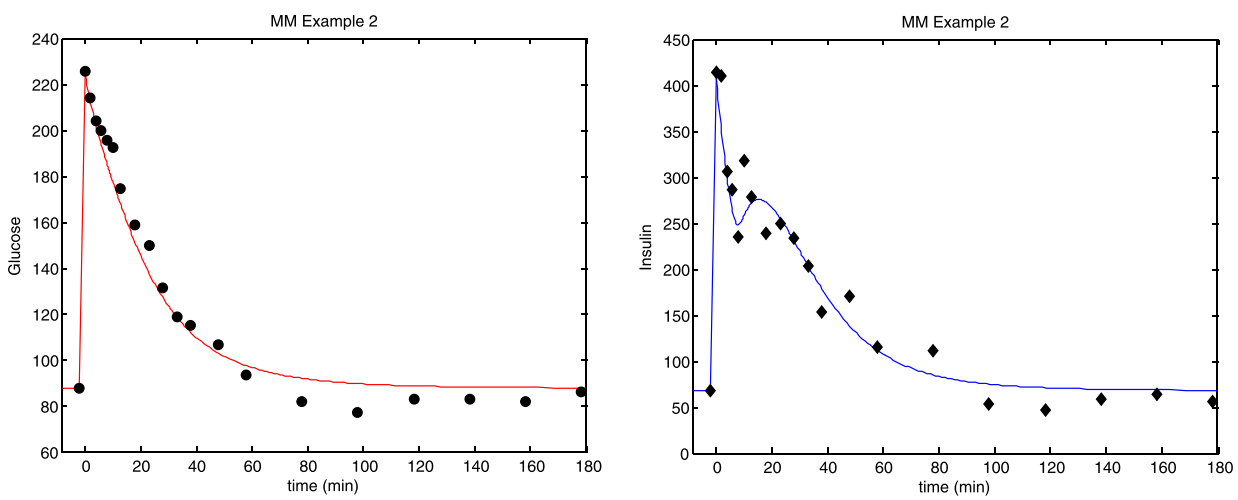

(a)
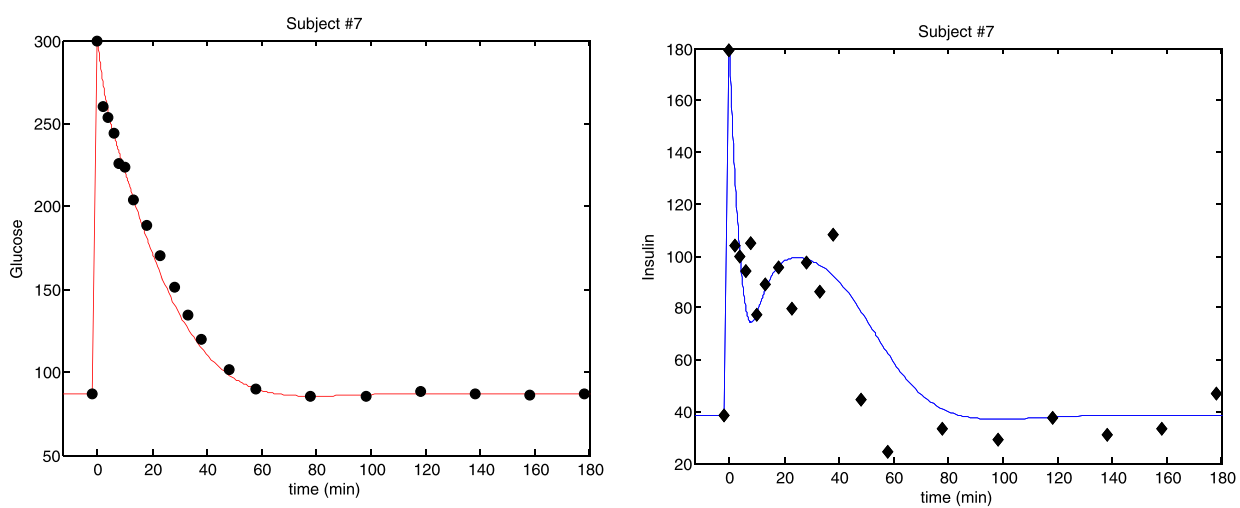

(b)
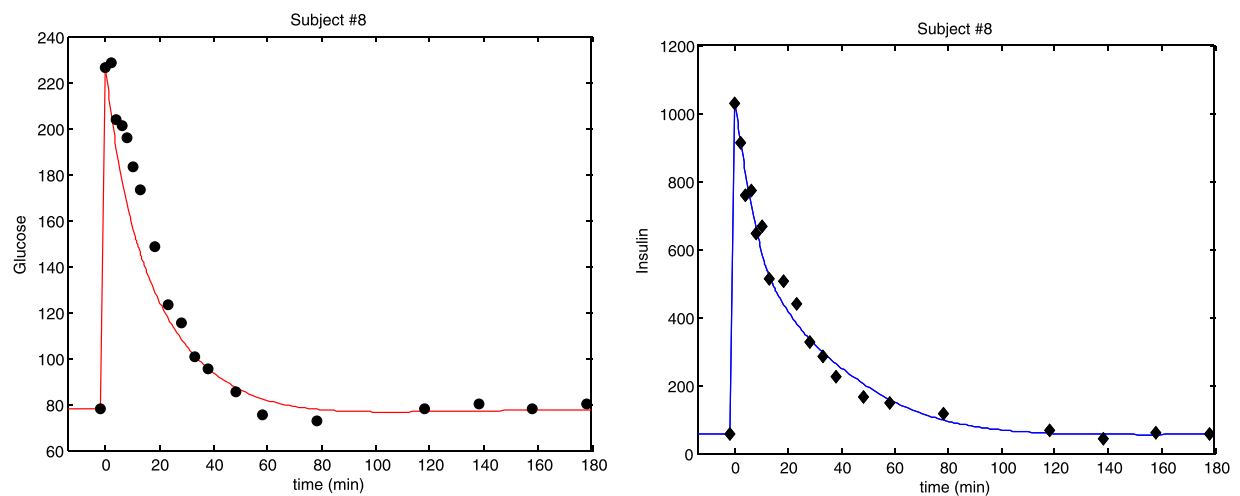

(c)
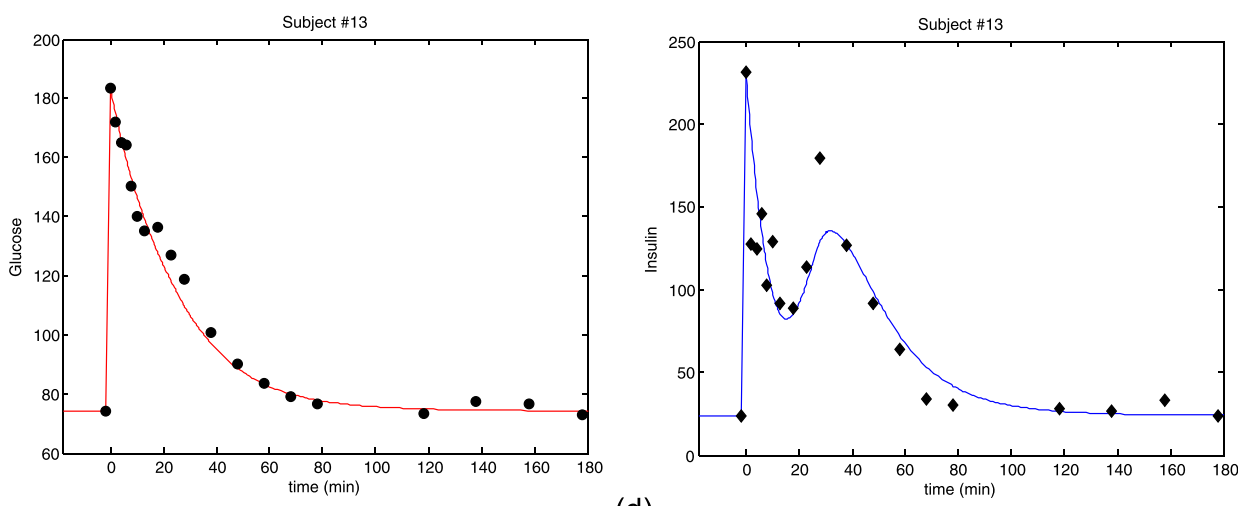

(d)

(if any) due to a new round of insulin secretion, in addition to other data points. While the initial condition of the insulin concentration $I$ is set at $I(0)$, the initial condition of the glucose concentration $G$ is given by (1.7) with fixed value $\delta=2$ (min) in observation that the bolus of glucose is injected within two minutes in IVGTT. Every parameter has its
FIG. 2. Simulation profiles obtained by Model (1.3) for subject nos. 6, 7, 8, and 13. Estimated parameter values are in Table I. The dots and diamonds are glucose data and insulin data, respectively. The curves are model profiles. physiological meaning, and thus, their values are positive. On the other hand, when consider them in the context of mathematics, any parameter value can be negative or zero, which would result in the parameter values do not reflect physiological characteristics. To avoid this, we set the constraints for the parameters in positive intervals. 


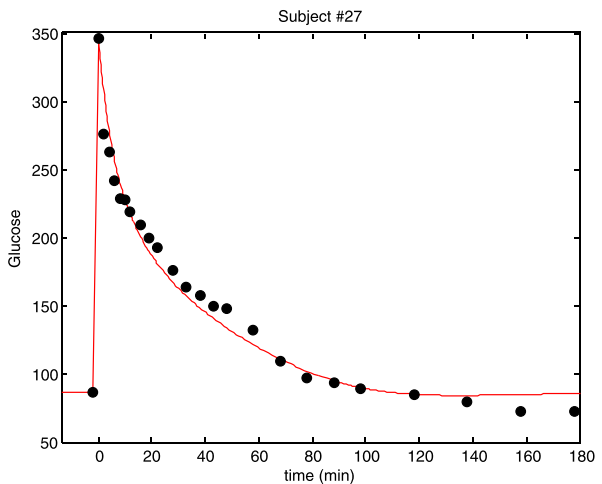

(a)

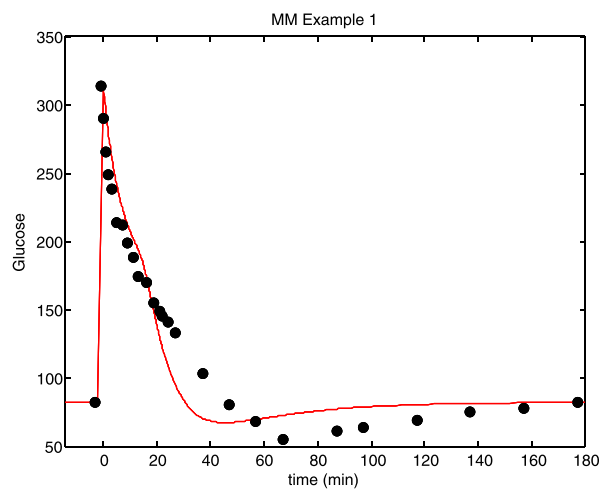

(c)

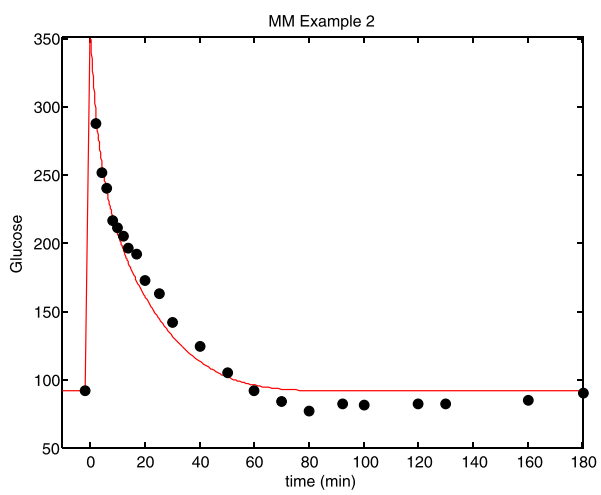

(e)

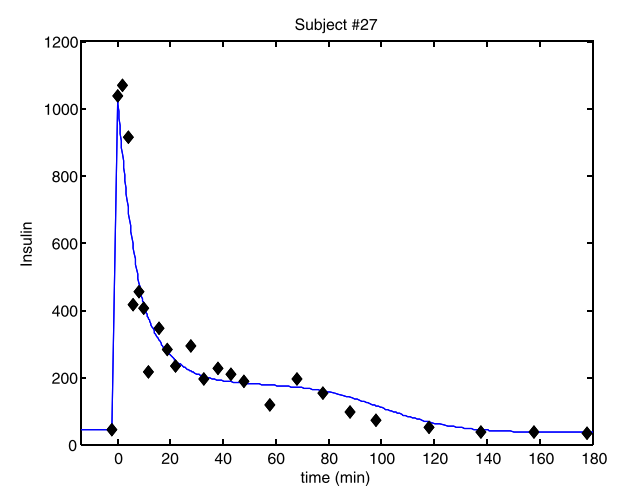

(b)

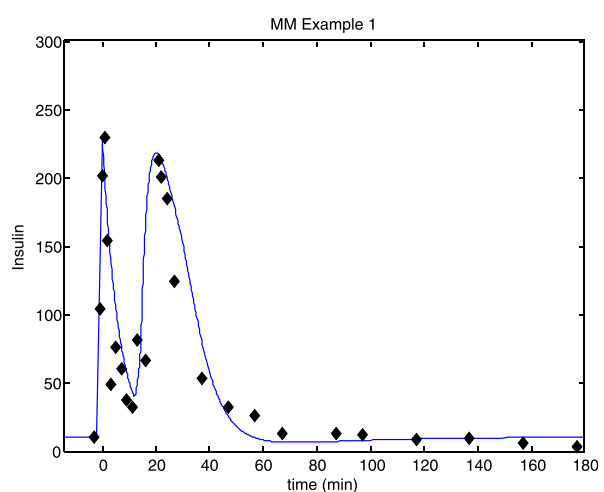

(d)

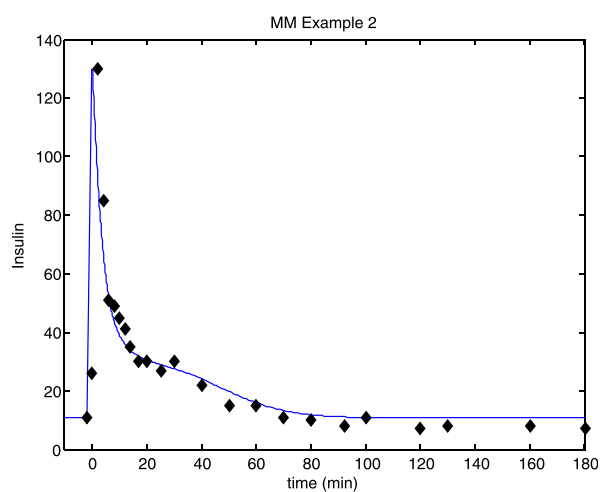

(f)
FIG. 3. Simulation profiles obtained by Model (1.3) for subject no. 27, Penn Millennium Ex 1 and Ex 2. Estimated parameter values are in Table II. The dots and diamonds are glucose data and insulin data, respectively. The curves are model profiles.

TABLE III. Comparison between the values of parameter $S_{g}, S_{i}$, and delay parameters estimated by Model (1.3), Penn Millennium MM ${ }^{12}$ and Model(1) in Ref. 26.

\begin{tabular}{|c|c|c|c|c|}
\hline Subjects & Model & $S_{g}$ & $\mathrm{~S}_{\mathrm{i}}$ & Delay \\
\hline \multirow[t]{2}{*}{ MM Ex 1} & Model(1.3) & $2.0600 \times 10^{-02}$ & $2.4930 \times 10^{-04}$ & {$\left[-1.4566 \times 10^{+01},-1.3566 \times 10^{+01}\right]$} \\
\hline & Minimal Model $^{12}$ & $2.4594 \times 10^{-02}$ & $2.8526 \times 10^{+00}$ & $\ldots$ \\
\hline \multirow[t]{2}{*}{ MM Ex 2} & Model(1.3) & $8.2897 \times 10^{-03}$ & $7.5621 \times 10^{-04}$ & {$\left[-1.5066 \times 10^{+01},-5.0661 \times 10^{+00}\right]$} \\
\hline & Minimal Model ${ }^{12}$ & $9.5426 \times 10^{-03}$ & $7.4555 \times 10^{+00}$ & \\
\hline \multirow[t]{2}{*}{ \#6 } & Model(1.3) & $1.6150 \times 10^{-02}$ & $5.4371 \times 10^{-05}$ & {$\left[-1.0250 \times 10^{+01},-6.2500 \times 10^{+00}\right]$} \\
\hline & 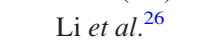 & $6.5973 \times 10^{-03}$ & $6.7604 \times 10^{-05}$ & $8.0662 \times 10^{+00}$ \\
\hline \multirow[t]{2}{*}{$\# 7$} & Model(1.3) & $2.1810 \times 10^{-02}$ & $2.0191 \times 10^{-04}$ & {$\left[-1.7369 \times 10^{+01},-7.3688 \times 10^{+00}\right]$} \\
\hline & $\mathrm{Li}$ et al. ${ }^{26}$ & $3.6921 \times 10^{-04}$ & $1.0081 \times 10^{-06}$ & $1.0169 \times 10^{+01}$ \\
\hline \multirow[t]{2}{*}{$\# 8$} & Model(1.3) & $1.5998 \times 10^{-02}$ & $3.5585 \times 10^{-05}$ & {$\left[-1.7250 \times 10^{+01},-1.1250 \times 10^{+01}\right]$} \\
\hline & $\mathrm{Li}$ et $a .^{26}$ & $1.1990 \times 10^{-02}$ & $3.0209 \times 10^{-05}$ & $5.3818 \times 10^{+00}$ \\
\hline \multirow[t]{2}{*}{$\# 13$} & Model(1.3) & $3.5000 \times 10^{-02}$ & $2.3000 \times 10^{-05}$ & {$\left[-2.7000 \times 10^{+01},-9.0000 \times 10^{+00}\right]$} \\
\hline & Li et al. ${ }^{26}$ & $8.7969 \times 10^{-03}$ & $1.1761 \times 10^{-04}$ & $1.8000 \times 10^{+01}$ \\
\hline \multirow[t]{2}{*}{$\# 27$} & Model(1.3) & $1.3630 \times 10^{-03}$ & $6.2029 \times 10^{-05}$ & {$\left[-1.6914 \times 10^{+01},-1.0914 \times 10^{+01}\right]$} \\
\hline & Li et $_{\text {al. }}{ }^{26}$ & $2.9076 \times 10^{-05}$ & $6.3829 \times 10^{-05}$ & $3.0000 \times 10^{+00}$ \\
\hline
\end{tabular}




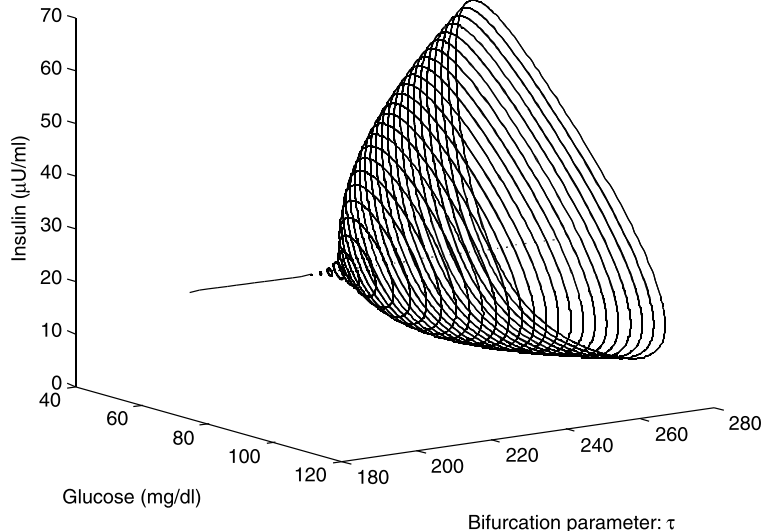

(a)

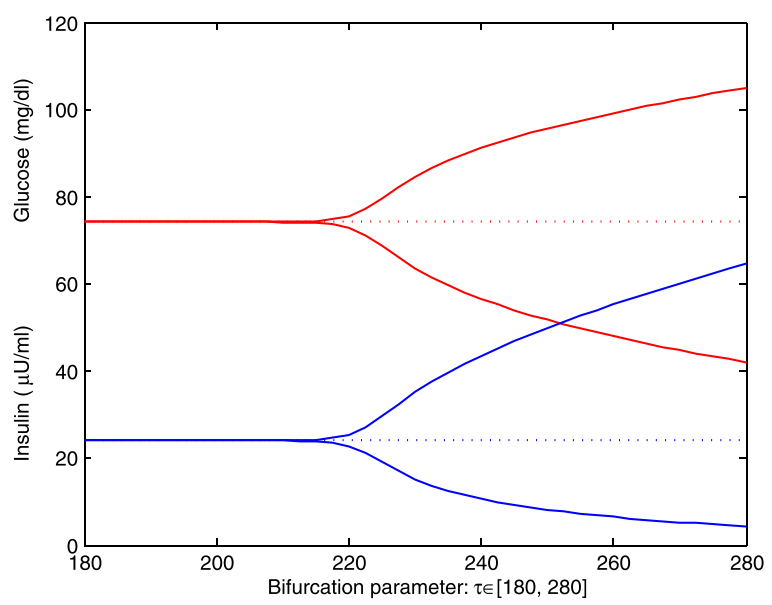

(b)

FIG. 4. The emerged limit cycles of the parameter $\tau \in[180,280]$ while $\epsilon=5$ for the subject \#13 in Ref. 24 produced by Model (1.3) with parameters $S_{i}=$ $0.00015, b=0.41552, d_{i}=0.08, \sigma=39.5193, S_{g}=0.002, \alpha=200$ and $n=3.1$.

We have analytically shown the existence of Hopf bifurcation when the delay parameter $\tau$ increases from small, and $\epsilon$ decreases from large, respectively. Taking the subject no. 13 as an example, we demonstrated the bifurcation in Fig. 4 when $\tau$ increases, and in Fig. 5 when $\epsilon$ decreases, respectively, while all other parameter values are fixed. Figure 4 indicates that a Hopf bifurcation takes place at $\tau_{0} \approx 211$, while Fig. 5 indicates that a Hopf bifurcation takes place when $\epsilon_{0} \approx 110$. Apparently, in such cases, the delay parameter values are out of physiological meaningful range. That is to say, with physiological meaningful parameter values, Model (1.3) predicts that the equilibrium is stable.

\section{DISCUSSION}

It is known that MM overestimates the glucose effectiveness index $S_{g}{ }^{15,16}$ The estimated values of $S_{g}$ by our model (1.3) are more reasonable than those obtained by MM and the models in Refs. 24 and 26.This is achieved through better model fitting to the data set by adjusting the two parameters $\tau$ and $\epsilon$, which together describe the insulin secretion time delay interval.

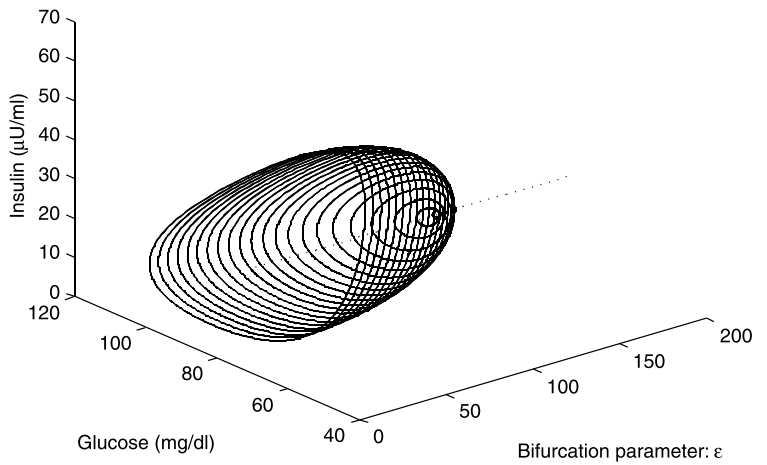

(a)

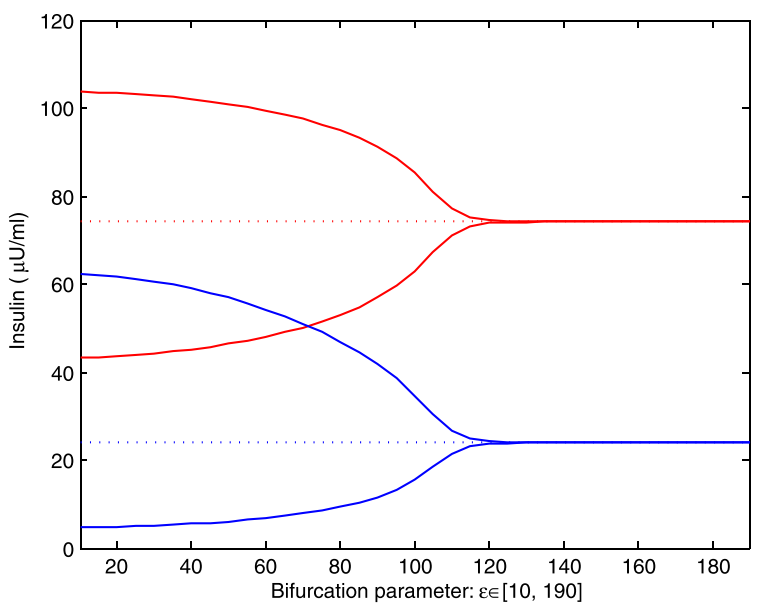

(b)

FIG. 5. The emerged limit cycles of the parameter $\epsilon \in[10,190]$ with $\tau=275$ for the subject no. 13 in Ref. 24 produced by Model (1.3) with parameters $S_{i}=0.00015, b=0.41552, d_{i}=0.08, \sigma=39.5193, S_{g}=0.002$, $\alpha=200$ and $n=3.1$.

Often, larger time delay in a delay differential equation system destabilizes the system through a Hopf bifurcation. The delayed effect of insulin secretion in response to the rapidly elevated glucose level is normally ranged in a few minutes to less than half of an hour. Our numerical simulations show that the Model (1.3) remains asymptotically stable for the delay value in physiological range. The Hopf bifurcation does not occur if the delay center is smaller than two hours. This indicates that in a typical clinical setting, the destabilization will not be a concern and the dynamics of glucose and insulin will approach their basal levels within three hours.

In this paper, we propose a novel approach to model the delayed effect in the past sub-interval centered at $t-\tau$ with radius $\epsilon$. Such approach is also applicable to other disciplines. Our analytical analysis and numerical study show that the delay center plays the major role and the delay radius takes the secondary role. We summarize our main mathematical findings as follows:

(A) when the delay center is fixed, the smaller the radius of the distribution interval of the delay, the more likely the system could be destabilized; 
(B) for a certain and finite time horizon, when the radius of the delay interval is fixed, the farther away of the delay center from the current time, the more likely the system could be destabilized.

We point out that the delay over the past sub-interval can be realized by selecting the kernel

$$
\omega(\theta)= \begin{cases}\frac{1}{2 \epsilon}, & \theta \in[\tau-\epsilon, \tau+\epsilon] \\ 0, & \text { otherwise }\end{cases}
$$

in the general delay form over the full past interval

$$
\int_{0}^{\tau+\epsilon} \omega(\theta) G(t-\theta) d \theta
$$

Minimal Model (MM) received its name since it has only three parameters, the minimal number of parameters in all well known IVGTT models. Model (1.3) has six parameters (notice that the parameter $b$ is solvable by assuming its equilibrium point at the basal levels of glucose and insulin concentrations, respectively). If one model, say model 1 , contains all the parameters of another model, say model 2, and the model 1 becomes model 2 when parameters not used in model 2 are set to be zero, then one can expect that model 1 shall fit data at least as good as model 2. Otherwise, more parameters in a model may not ensure better data fitting. In
Everett et $a{ }^{28}$ and in Rutter et al., ${ }^{29}$ the authors reported that simpler ad hoc models actually fit data slightly better than their more complex models. However, Everett et al. ${ }^{28}$ found that their more complex and realistic models do provide better predictions.

\section{ACKNOWLEDGMENTS}

Xiangyun Shi is supported by Chinese NNSF 11701495. The work of Jiaxu Li was partially supported by the DOE Grant No. DE-EM0000197 and Chinese NNSF 11701495. The work of Yang Kuang and Athena Makroglou was partially supported by the London Mathematical Society (LMS) Grant Scheme 4, No. 41230, which financed the visit of Yang Kuang to the University of Portsmouth in January 2014. The work of Yang Kuang is also partially supported by NSF Grant Nos. DMS-1518529 and DMS-1615879.

\section{APPENDIX A: PROOF OF THEOREM 4.2}

Proof. We need only to show that the conjugate imaginary roots of (4.1) cross the imaginary axis from right to left, that is,

$$
\left.\frac{d R e(\lambda)}{d \tau}\right|_{\lambda=i \hat{\omega}}>0
$$

From (4.1), we have

$$
\frac{d \lambda}{d \tau}\left(2 \lambda+a-\frac{h}{2 \epsilon \lambda^{2}}\left(e^{(-\tau+\epsilon) \lambda}-e^{(-\tau-\epsilon) \lambda}\right)\right)+\frac{h}{2 \epsilon \lambda}\left(e^{(-\tau+\epsilon) \lambda}(-\tau+\epsilon)-e^{(-\tau-\epsilon) \lambda}(-\tau-\epsilon)\right)=\frac{h}{2 \epsilon}\left(e^{(-\tau+\epsilon) \lambda}-e^{(-\tau-\epsilon) \lambda}\right)
$$

Hence,

$$
\left(\frac{d \lambda}{d \tau}\right)^{-1}=\frac{2 \lambda+a-\frac{h}{2 \epsilon \lambda^{2}}\left(e^{(-\tau+\epsilon) \lambda}-e^{(-\tau-\epsilon) \lambda}\right)+\frac{h}{2 \epsilon \lambda}\left(e^{(-\tau+\epsilon) \lambda}(-\tau+\epsilon)-e^{(-\tau-\epsilon) \lambda}(-\tau-\epsilon)\right)}{\frac{h}{2 \epsilon}\left(e^{(-\tau+\epsilon) \lambda}-e^{(-\tau-\epsilon) \lambda}\right)} .
$$

At $\lambda=i \hat{\omega}$, we have

$$
\begin{aligned}
\operatorname{sgn}\left\{\left.\left(\frac{d R e(\lambda)}{d \tau}\right)\right|_{\lambda=i \hat{\omega}}\right\}= & \operatorname{sgn}\left\{\left.\left(\frac{d R e(\lambda)}{d \tau}\right)^{-1}\right|_{\lambda=i \hat{\omega}}\right\}=\operatorname{sgn}\left\{\operatorname{Re}\left(\left.\left(\frac{d \lambda}{d \tau}\right)^{-1}\right|_{\lambda=i \hat{\omega}}\right)\right\} \\
= & \operatorname{sgn}\left\{\frac{2 \hat{\omega}((\sin (-\tau+\epsilon)) \hat{\omega}-\sin ((-\tau-\epsilon) \hat{\omega}))+a(\cos ((-\tau+\epsilon) \hat{\omega})-\cos ((-\tau-\epsilon) \hat{\omega}))}{\frac{1}{2 \epsilon}\left[(\cos ((-\tau+\epsilon) \hat{\omega})-\cos ((-\tau-\epsilon) \hat{\omega}))^{2}+\left(\sin ((-\tau+\epsilon) \hat{\omega})-\sin ((-\tau-\epsilon) \hat{\omega})^{2}\right]\right.}\right. \\
& +\frac{\frac{h}{2 \epsilon \hat{\omega}^{2}}\left(2-2 \cos ((-\tau+\epsilon) \hat{\omega}) \cos ((-\tau-\epsilon) \hat{\omega})-2 \sin ((-\tau+\epsilon) \hat{\omega}) \sin ((-\tau-\epsilon) \hat{\omega})-\frac{h}{\hat{\omega}} \sin (2 \epsilon \hat{\omega})\right)}{=} \\
= & \operatorname{sgn}\left\{\hat{\omega} \cos (-\tau \hat{\omega}) \sin (\epsilon \hat{\omega})-2 \sin (-\tau \hat{\omega}) \sin (\epsilon \hat{\omega}) a+\frac{h}{2 \epsilon \hat{\omega}^{2}}(2-2 \cos (2 \epsilon \hat{\omega}))-\frac{h}{\hat{\omega}} \sin (2 \epsilon \hat{\omega})\right\} \\
= & \operatorname{sgn}\left\{4 \hat{\omega}^{2} \frac{\left(\hat{\omega}^{2}-c\right) \hat{\omega} \epsilon}{h}+2 a \frac{a \epsilon \hat{\omega}^{2}}{h}+\frac{h}{\epsilon \hat{\omega}^{2}}(1-\cos (2 \epsilon \hat{\omega}))-\frac{h}{\hat{\omega}} \sin (2 \epsilon \hat{\omega})\right\} \\
= & \operatorname{sgn}\left\{\frac{4 \hat{\omega}^{4}}{h} \epsilon^{2}+\frac{2 \epsilon \hat{\omega}^{2}}{h}\left(a^{2}-2 c\right)+\frac{2 h}{\hat{\omega}^{2}} \sin (\epsilon \hat{\omega})\left(\frac{\sin (\epsilon \hat{\omega})}{\epsilon \hat{\omega}}-\cos (\epsilon \hat{\omega})\right)\right\}=1 \text { for } 0<\hat{\omega}<\frac{\pi}{\epsilon},
\end{aligned}
$$


which is ensured by $a^{2}-2 c>0$ and $\sin x-x \cos x>0$ for $0<x<\pi$. Therefore, (1.3) undergoes a bifurcation when $\tau=$ $\frac{1}{\hat{\omega}} \cot ^{-1}\left(\frac{\hat{\omega}^{2}-c}{a \hat{\omega}}\right)$ for $0<\hat{\omega}<\frac{\pi}{\epsilon}$.

\section{APPENDIX B: PROOF OF THEOREM 4.4}

Proof. Again, we need only to show that

$$
\left.\frac{d \operatorname{Re}(\lambda)}{d \epsilon}\right|_{\lambda=i \hat{\omega}^{*}}<0
$$

From (4.1), we can get that

$$
2 \epsilon \lambda^{3}+2 \epsilon a \lambda^{2}+2 \epsilon c \lambda+h\left(e^{(-\tau+\epsilon) \lambda}-e^{(-\tau-\epsilon) \lambda}\right)=0, \text { for } \lambda \neq 0 .
$$

Hence

$$
\left(\frac{d \lambda}{d \epsilon}\right)^{-1}=\frac{6 \epsilon \lambda^{2}+4 a \epsilon \lambda+h e^{(-\tau+\epsilon) \lambda}(-\tau+\epsilon)+h e^{(-\tau-\epsilon) \lambda}(\tau+\epsilon)+2 c \epsilon}{-2 \lambda^{3}-2 a \lambda^{2}-h \lambda\left(e^{(-\tau+\epsilon) \lambda}+e^{(-\tau-\epsilon) \lambda}\right)-2 \lambda c},
$$

and thus, at $\lambda=i \omega^{*}$,

$$
\left.\left(\frac{d \lambda}{d \epsilon}\right)^{-1}\right|_{\lambda=i \hat{\omega}^{*}}=\frac{P}{Q}
$$

where

$$
\begin{gathered}
P=-6 \epsilon \omega^{* 2}+2 \epsilon c+h\left(\cos \left((-\tau+\epsilon) \omega^{*}\right)(-\tau+\epsilon)+\cos \left((-\tau-\epsilon) \omega^{*}\right)(\tau+\epsilon)\right) \\
+i\left(4 a \epsilon \omega^{*}+h\left(\sin \left((-\tau+\epsilon) \omega^{*}\right)(-\tau+\epsilon)+\sin \left((-\tau-\epsilon) \omega^{*}\right)(\tau+\epsilon)\right)\right), \\
Q=2 a \omega^{* 2}+h \omega^{*}\left(\sin \left((-\tau+\epsilon) \omega^{*}\right)+\sin \left((-\tau-\epsilon) \omega^{*}\right)\right)+i\left(2 \omega^{* 3}-2 c \omega^{*}-h \omega^{*}\left(\cos \left((-\tau+\epsilon) \omega^{*}\right)+\cos \left((-\tau-\epsilon) \omega^{*}\right)\right)\right) .
\end{gathered}
$$

Therefore

$$
\begin{aligned}
\operatorname{sgn}\left\{\operatorname{Re}\left\{\left.\left(\frac{d \lambda}{d \epsilon}\right)^{-1}\right|_{\lambda=i \omega^{*}}\right\}\right\}= & \operatorname{sgn}\left\{-4 a \epsilon \omega^{* 4}-4 a c \epsilon \omega^{* 2}+\left(-2 a \tau h \omega^{* 2}-2 a \epsilon h \omega^{* 2}\right) \cos \left((-\tau+\epsilon) \omega^{*}\right)\right. \\
& +\left(2 a \tau h \omega^{* 2}-2 a \epsilon h \omega^{* 2}\right) \cos \left((-\tau-\epsilon) \omega^{*}\right)+\left(-4 \epsilon h \omega^{* 3}-2 h \tau \omega^{* 3}+2 c h \tau \omega^{*}\right) \sin \left((-\tau+\epsilon) \omega^{*}\right) \\
& +\left(-4 \epsilon h \omega^{* 3}+2 h \tau \omega^{* 3}-2 c h \tau \omega^{*}\right) \sin \left((-\tau-\epsilon) \omega^{*}\right)+2 \tau \omega^{*} h \sin \left(2 \epsilon \omega^{*}\right) \\
= & \operatorname{sgn}\left\{-4 a c \omega^{* 4}-4 a c \epsilon \omega^{* 2}+\left(-2 a \tau h \omega^{* 2}-2 a \epsilon h \omega^{* 2}\right) \cos \left(\tau \omega^{*}\right) \cos \left(\epsilon \omega^{*}\right)\right. \\
& +\left(-2 a \tau h \omega^{* 2}-2 a \epsilon h \omega^{* 2}\right) \sin \left(\tau \omega^{*}\right) \sin \left(\epsilon \omega^{*}\right)+\left(2 a \tau h \omega^{* 2}-2 a \epsilon h \omega^{* 2}\right) \cos \left(\tau \omega^{*}\right) \cos \left(\epsilon \omega^{*}\right) \\
& -\left(2 a \tau h \omega^{* 2}-2 a \epsilon h \omega^{* 2}\right) \sin \left(\tau \omega^{*}\right) \sin \left(\epsilon \omega^{*}\right)-\left(-4 \epsilon h \omega^{* 3}-2 h \tau \omega^{* 3}+2 c \omega^{*} h \tau\right) \sin \left(\tau \omega^{*}\right) \cos \left(\epsilon \omega^{*}\right) \\
& +\left(-4 \epsilon h \omega^{* 3}-2 h \tau \omega^{* 3}+2 c \omega^{*} h \tau\right) \cos \left(\tau \omega^{*}\right) \sin \left(\epsilon \omega^{*}\right)-\left(-4 \epsilon h \omega^{* 3}-2 h \tau \omega^{* 3}+2 c \omega^{*} h \tau\right) \\
& \left.\times \sin \left(\tau \omega^{*}\right) \cos \left(\epsilon \omega^{*}\right)-\left(-4 \epsilon h \omega^{* 3}-2 h \tau \omega^{* 3}+2 c \omega^{*} h \tau\right) \cos \left(\tau \omega^{*}\right) \sin \left(\epsilon \omega^{*}\right)+2 \tau \omega^{*} h \sin \left(2 \epsilon \omega^{*}\right)\right\} \\
= & \operatorname{sgn}\left\{-4 a c \omega^{* 4}-4 a c \epsilon \omega^{* 2}+\left(-4 a \epsilon h \omega^{* 2}\right) \cos \left(\tau \omega^{*}\right) \cos \left(\epsilon \omega^{*}\right)+\left(-4 a \epsilon h \omega^{* 2}\right) \sin \left(\tau \omega^{*}\right) \sin \left(\epsilon \omega^{*}\right)\right. \\
& \left.+8 \epsilon h \omega^{* 3} \sin \left(\tau \omega^{*}\right) \cos \left(\epsilon \omega^{*}\right)+\left(-4 \epsilon h \omega^{* 3}+4 c \epsilon h \omega^{*}\right) \cos \left(\tau \omega^{*}\right) \sin \left(\epsilon \omega^{*}\right)+2 \tau \omega^{*} h \sin \left(2 \epsilon \omega^{*}\right)\right\} .
\end{aligned}
$$

From (4.2), we can get

$$
\sin \left(\tau \omega^{*}\right) \sin \left(\epsilon \omega^{*}\right)=\frac{a \epsilon \omega^{* 2}}{h} \text { and } \cos \left(\tau \omega^{*}\right) \sin \left(\epsilon \omega^{*}\right)=\frac{\left(\omega^{* 2}-c\right) \epsilon \omega^{* 2}}{h},
$$

and

$$
\sin \left(\tau \omega^{*}\right)=\frac{a \epsilon \omega^{* 2}}{h \sin \left(\epsilon \omega^{*}\right)} \text { and } \cos \left(\tau \omega^{*}\right)=\frac{\left(\omega^{* 2}-c\right) \epsilon \omega^{*}}{h \sin \left(\epsilon \omega^{*}\right)} .
$$

So, we finally have 


$$
\begin{aligned}
\operatorname{sgn}\left\{\left.\left(\frac{d \operatorname{Re}(\lambda)}{d \epsilon}\right)^{-1}\right|_{\lambda=i \omega^{*}}\right\}= & \operatorname{sgn}\left\{\operatorname{Re}\left\{\left.\left(\frac{d \lambda}{d \epsilon}\right)^{-1}\right|_{\lambda=i \omega^{*}}\right\}\right\}=\operatorname{sgn}\left\{-4 a c \omega^{* 4}-4 a c \epsilon \omega^{* 2}-4 a^{2} \tau \epsilon \omega^{* 4}+\left(4 a \epsilon^{2} \omega^{* 5}+4 a \epsilon^{2} \omega^{* 3} c\right)\right. \\
& \left.\times \frac{\cos \left(\epsilon \omega^{*}\right)}{\sin \left(\epsilon \omega^{*}\right)}-4 \tau \epsilon \omega^{* 4}\left(\omega^{* 2}-c\right)^{2}+4 \tau \omega^{*} h^{2} \sin \left(\epsilon \omega^{*}\right) \cos \left(\epsilon \omega^{*}\right)\right\}=-1, \quad \text { for } \frac{\pi}{2}<\epsilon \omega^{*}<\pi
\end{aligned}
$$

${ }^{1}$ Centers for Disease Control and Prevention, National Diabetes Statistics Report: Estimates of Diabetes and Its Burden in the United States, 2014 (Department of Health and Human Services, Centers for Disease Control and Prevention, Atlanta, GA, USA, 2014).

${ }^{2}$ S. E. Kahn, M. E. Cooper, and S. Del Prato, "Pathophysiology and treatment of type 2 diabetes: Perspectives on the past, present, and future," Lancet 383(9922), 1068-1083 (2014).

${ }^{3}$ M. A. Abdul-Ghani, D. Tripathy, and R. A. DeFronzo, "Contributions of beta-cell dysfunction and insulin resistance to the pathogenesis of impaired glucose tolerance and impaired fasting glucose," Diabetes Care 29(5), 1130-1139 (2006).

${ }^{4}$ D. A. Lang, D. R. Matthews, and R. C. Tuner, "Brief, irregular oscillations of basal plasma insulin and glucose concentrations in diabetic man," Diabetes 30, 435-439 (1981).

${ }^{5}$ L. S. Satin, P. C. Butler, J. Ha, and A. S. Sherman, "Pulsatile insulin secretion, impaired glucose tolerance and type 2 diabetes," Mol. Aspects Med. 42, 61-77 (2015).

${ }^{6} \mathrm{~S}$. O'Rahilly, R. C. Turner, and D. R. Matthews, "Impaired pulsatile secretion of insulin in relatives of patients with non-insulin-dependent diabetes," N. Engl. J. Med. 318(19), 1225-1230 (1988).

${ }^{7}$ M. B. Egede, J. E. Henriksen, T. T. Durck, K. Levin, C. Rantzau, G. Ward, H. Beck-Nielsen, and F. P. Alford, "Glucose effectiveness in nondiabetic relatives: Dysglycemia and $\beta$-cell function at 10 years," J. Clin. Endocrinol. Metab. 99(4), 1420-1424 (2014).

${ }^{8}$ R. Muniyappa, S. Lee, H. Chen, and M. J. Quon, "Current approaches for assessing insulin sensitivity and resistance in vivo: Advantages, limitations, and appropriate usage," Am. J. Physiol. Endocrinol. Metab. 294(1), E15-E26 (2008).

${ }^{9}$ R. N. Bergman and C. Cobelli, "Minimal modeling/partition analysis and the estimation of insulin sensitivity," Fed. Proc. 39, 110-115 (1980).

${ }^{10}$ R. N. Bergman, Y. Z. Ider, C. R. Bowden, and C. Cobelli, "Quantitative estimation of insulin sensitivity,” Am. J. Physiol. 236, E667-E677 (1979).

${ }^{11}$ E. Ferrannini and A. Mari, "How to measure insulin sensitivity," J. Hypertens. 16, 895-906 (1998).

${ }^{12}$ See http://research.vet.upenn.edu/biomath/currentprojects/diabetesglucosemetabolism/tabid/1622/default.aspx; https://www.cedars-sinai.edu/ Research/Research-Labs/Bergman-Lab/Reagents-and-Resources.aspx for MINMOD Millennium Software.

${ }^{13}$ R. C. Boston, D. Stefanovski, P. J. Moate, A. E. Sumner, R. M. Watanabe, and R. N. Bergman, "MINMOD Millennium: A computer program to calculate glucose effectiveness and insulin sensitivity from the frequently sampled intravenous glucose tolerance test," Diabetes Technol. Ther. 5(6), 1003-1015 (2003).

${ }^{14}$ R. N. Bergman, "Minimal model: Perspective from 2005," Horm. Res. 64(3), 8-15 (2005).
${ }^{15}$ A. Mari, "Assessment of insulin sensitivity with minimal model: Role of model assumptions," Am. J. Physiol. 272(35), E925-E934 (1997).

${ }^{16}$ A. Caumo, P. Vicini, J. J. Zachwieja, A. Avogaro, K. Yarasheski, D. M. Bier, and C. Cobelli, "Undermodeling affects minimal model indexes: Insights from a two-compartment model," Am. J. Physiol. 276(39), E1171-E1193 (1999).

${ }^{17} \mathrm{~J}$. Li, Y. Kuang, and C. Mason, "Modeling the glucose and insulin regulatory system and ultradian insulin secretory oscillations with two time delays," J. Theor. Biol. 242, 722-735 (2006).

${ }^{18}$ A. De Gaetano and O. Arino, "Mathematical modeling of the intravenous glucose tolerance test," J. Math. Biol. 40, 136-168 (2000).

${ }^{19} \mathrm{~J}$. Li and Y. Kuang, "Analysis of a model of the glucose-insulin regulatory system with two delays," SIAM J. Appl. Math. 67, 757-776 (2007).

${ }^{20}$ A. Makroglou, J. Li, and Y. Kuang, "Mathematical models and software tools for the glucose and insulin regulatory system and diabetes: An overview," Appl. Numer. Math. 56, 559-573 (2006).

${ }^{21}$ D. L. Bennett and S. A. Gourley, "Global stability in a model of the glucose-insulin interaction with time delay,” Eur. J. Appl. Math. 15, 203-221 (2004).

${ }^{22}$ P. Palumbo, S. Panunzi, and A. D. Gaetano, "Qualitative behavior of a family of delay-differential models of the glucose-insulin system," Discrete Contin. Dyn. Syst., Ser. B 7, 399-424 (2007).

${ }^{23} \mathrm{~J}$. Li, Y. Kuang, and B. Li, "Analysis of IVGTT glucose insulin interaction models with time delay," Discrete Contin. Dyn. Syst., Ser. B 1(1), 103-124 (2001).

${ }^{24}$ S. Panunzi, P. Palumbo, and A. De Gaetano, "A discrete single delay model for the intravenous glucose tolerance test," Theor. Biol. Med. Mod. 4, 35-31 (2007).

${ }^{25}$ S. Panunzi, A. De Gaetano, and G. Mingrone, "Advantages of the single delay model for the assessment of insulin sensitivity from the intra-venous glucose tolerance test," Theor. Biol. Med. Model. 7(1), 9 (2010).

${ }^{26}$ J. Li, M. Wang, A. De Gaetano, P. Palumbo, and S. Panunzi, "The range of time delay and the global stability of the equilibrium for an IVGTT model," Math. Biosci. 235, 128-137 (2012).

${ }^{27}$ J. Sturis, K. S. Polonsky, E. Mosekilde, and E. Van Cauter, "Computer model for mechanisms underlying ultradian oscillations of insulin and glucose," Am. J. Physiol. 260, E801-E809 (1991).

${ }^{28}$ R. A. Everett, A. Packer, and Y. Kuang, "Can mathematical models predict the outcomes of prostate cancer patients undergoing intermittent androgen deprivation therapy?," Biophys. Rev. Lett. 9, 173-191 (2014).

${ }^{29}$ E. M. Rutter, H. T. Banks, G. A. LeBlanc, and K. B. Flores, "Continuous structured population models for Daphnia magna," Bull. Math. Biol. 79(11), 2627-2648 (2017). 